\title{
Non-Starch Plant Polysaccharides: Physicochemical Modifications and Pharmaceutical Applications
}

\author{
Sutapa Biswas Majee*, Dhruti Avlani, Gopa Roy Biswas \\ Nshm College of Pharmaceutical Technology, Nshm Knowledge Campus, Kolkata-Group Of Institutions, 124 B L Saha Road, Kolkata-700 053, West \\ Bengal, India.
}

\section{ARTICLE INFO \\ Article history: \\ Received on: 24/05/2016 \\ Revised on: 21/06/2016 \\ Accepted on: 24/08/2016 \\ Available online: 29/10/2016}

Key words:

Non-starch polysaccharide, polymer blending, polymer grafting. gastroretentive, matrix tablet, mucoadhesive drug delivery, colon-specific drug delivery.

\begin{abstract}
Polysaccharides of natural, synthetic or semi-synthetic origin have been used from time immemorial in the development of drug delivery systems designed to achieve tailored and site-specific drug release. Starch-based polysaccharides derived from plants have been extensively studied in this regard. Natural polymeric excipients are preferred over their synthetic counterparts owing to their low cost, availability, biocompatibility, biodegradability and non-toxicity. The present review attempts to provide a new direction and a comprehensive insight on the physical properties, rheological behavior, toxicity profile, pharmaceutical applications, swelling behavior and drug diffusion kinetics from dosage forms based on non-starch polysaccharides of plant origin such as, psyllium, pectin, arabinoxylan, xyloglucan, guar gum galactomannan and konjac glucomannan. It has been observed from the current review that non-starch polysaccharides are safe for human consumption and can be successfully employed to deliver drugs specifically to stomach and colon in a sustained fashion. They have thus widened the scope of natural polymeric excipients and demand better industrial utilization on a commercial scale to minimize cost of production and to satisfy therapeutic needs in safe and effective manner.
\end{abstract}

\section{INTRODUCTION}

Polysaccharides are polymers comprised of monosaccharides linked together by $1 \rightarrow 2,1 \rightarrow 3,1 \rightarrow 4,1 \rightarrow 5$ and $1 \rightarrow 6$ glycosidic bonds in an $\alpha$ - or $\beta$-configuration. Monosaccharides with six carbons form the repeating units in the polymer skeleton. The general formula of polysaccharides is $\mathrm{C}_{\mathrm{x}}\left(\mathrm{H}_{2} \mathrm{O}\right)_{\mathrm{y}}$ where $\mathrm{x}$ is a number with values between 200 and 2500 (Namazi et al., 2010). Natural polysaccharides derived from plants find wide application in pharmaceutical field as viscosity enhancers, gelling agents, binders, disintegrants, superdisintegrants, matrix formers, rate controlling polymers and permeation enhancers in the formulation of liquid, semi-solid, solid, multiparticulate dosage forms and transdermal drug delivery system. They have been extensively employed to impart coating to the surface of the dosage form to achieve different

* Corresponding Author

Mailid:sutapabiswas2001@yahoo.co.in drug release patterns e.g pulsatile, sigmoidal, ideal zero-order kinetics. They are adaptable to chemical or biochemical modification and can be easily blended with synthetic or semisynthetic polymers (Ogaji et al., 2012; Namazi et al., 2012). Most of the natural polysaccharides are bioactive exhibiting different activities such as immunomodulation, stimulation of reticuloendothelial system, effect on haematopoietic system, blood sugar lowering, regulation of lipid metabolism, wound healing, anti-inflammatory, anti-oxidant, anti-virus and anti-tumor activities (Chen et al., 2012).

There have been several review articles on the use of natural polysaccharides such as alginates, chitosan, inulin, modified celluloses and starches and polysaccharides for colonic drug delivery but there has been none which provides a comprehensive review on natural non-starch polysaccharides, derived from plants and their specific applications in the design and development of hydrogels, multiparticulates, mucoadhesive drug delivery, gastroretentive drug delivery, colon-specific drug 
delivery, nasal delivery, matrix tablets and as tablet super disintegrant The present review article focuses on the properties of non-starch polysaccharides of plant-origin, their toxicity profile, properties of chemically modified, grafted and blended polymers, their myriad applications in fabrication of drug delivery systems with tailored and site-specific drug release, their swelling behavior and drug diffusion kinetics. The objective of the review is to impart a holistic knowledge and provide deep insight into characteristics and applications of natural non-starch polysaccharides.

\section{SOURCE, PROPERTIES AND TOXICTY PROFILE OF PLANT-DERIVED NON-STARCH POLYSACCHARIDES}

Polysaccharides are widespread in the plant kingdom and are found in cell walls, seed coat, exudates of stem, roots and legumes. Non-starch plant-derived polysaccharides highlighted in the article include psyllium, pectin, arabinoxylan, xyloglucan, guar galactomannan and konjac glucomannan.

Psyllium is obtained from dried ripe seeds of Plantago psyllium and $P$. indica whereas Ispagula husk is derived from the ripe seeds of Plantago ovata. It is utilized as a low-calorie food because of its high dietary fibre content, has been extensively used in therapeutic management of constipation, diarrhea, Crohn's disease and irritable bowel syndrome and has been investigated as bioactive polysaccharide showing probiotic activity. Chemically, the Ispaghula mucilage contains pentosan and aldobiouronic acid, which produces arabinose, galactose, galacturonic acid and rhamnose on hydrolysis (Hojjatoleslamy et al., 2013, Bindu et al., 2012). Presence of mixtures of soluble and insoluble fractions of psyllium fiber makes it a versatile additive for preparation of different types of pharmaceutical dosage forms (CarePsyllium website). Psyllium has been reported to be safe for all ages and for diabetic patients because of its positive effect on glycemic and lipid control (Singh, 2007; Psyllium website).

Pectins are anionic polysaccharides and abundant in the primary cell walls of dicotyledonous plants. They are composed of "smooth" regions contributed by the homogalacturonan (HG) and type I rhamnogalacturonan (RG-I) regions forming the "hairy" regions. Their average molecular weight varies between 50,000 and 1,80,000 since the number of building blocks may range from hundreds to around one thousand per single molecule (Li Fang et al., 2008). Polygalacturonic acids in the HG regions can be subjected to partial methyl esterification (Morris et al., 2010). Pectin has been found to be devoid of any adverse effect on hematological and histopathological parameters and biochemical values (WHO Food additive series No. 5).

Arabinoxylan is obtained from the cell walls of cereal endosperm like wheat bran, maize bran and ispagula husk. Its linear backbone consists of $\beta$ - $(1 \rightarrow 4)$-linked D-xylopyranosyl units comprising of $\alpha$-L-arabinofuranosyl substituents linked via $\mathrm{O}-2$ and/or O-2,3-positions of xylose units. The polysaccharides obtained from different cereals vary in their molecular weight, degree of substitution of arabinose, arabinose/xylose ratio (A/X ratio), distribution of arabinose residues and the content of ferulic acid. Ferulated arabinoxylans consist of few arabinose residues ester-linked on (O)-5 to ferulic acid (3-methoxy-4hydroxycinnamic acid) (Erum et al., 2015; Berlanga-Reyes et al., 2009). Psyllium arabinoxylan has been studied extensively for its medicinal, nutraceutical and pharmaceutical applications (Hussain et al., 2016). Arabinoxylan was found to be non-irritant to eyes and skin in rabbits (Erum et al., 2015).

Xyloglucan (XG) or galactoxyloglucan derived from the seed kernel of Tamarindus indica is designated as tamarind seed polysaccharide (TSP) and it is also obtained from seeds of Copaifera langsdorffii, Hymenaea courbaril (Arruda et al., 2015). It possesses several desirable characteristics for its use in different types of pharmaceutical dosage forms. These properties include high viscosity, swelling and binding abilities, tolerance to wide range of $\mathrm{pHs}$, insensitivity to presence of salts, biocompatibility, high drug entrapment efficiency, high thermal stability and finally mucoadhesivity (Madgulkar et al., 2013). Xyloglucan is a hemicellulose with main cellulosic structure intact. It is structurally composed of $\beta$-1,4-linked glucose residues with the chain length varying between 300 to 3000 glucose units, most of which are substituted with 1-6 linked xylose side chains. TSP consists of three units of xyloglucan oligomers along with hexasaccharides, octasaccharides and nonasaccharides and its molecular weight of TSP varies from 650,000 to 2,500,000, which is higher compared to other wood-derived hemicelluloses. High percentage of galactose units in the molecule accounts for its neutral nature (Bach, 2014; Arruda et al., 2015). Although, it is water-soluble, its individual chains remain partially hydrated. The equilibrium moisture content is reported to be lower than that of starches up to relative humidity values of $70 \%$, probably due to cellulosic backbone in the molecular structure (Jose, 2012). Xyloglucan is reported to be non-toxic, non-irritant to ocular tissues and non-hemolytic (Nussinovitch, 2010; Mahajan and Desmukh, 2015; Arruda et al., 2015).

Galactomannans are widely distributed in the plant kingdom and are usually available from the endosperm cell wall of the seeds as storage polysaccharide. Chemically, they consist of $(1 \rightarrow 4)$-linked $\alpha$-D-mannopyranosyl backbone, with substitutions at O- 6 by ingle units of $\beta$-D-galactopyranose. Solubility of the polysaccharides depends on mannose/galactose ratio, which again varies with the source. Higher galactose content accounts for higher aqueous solubility. They have found uses in pharmaceutical formulations as emulsion stabilizers, thickening agents and viscosity-building agents. Guar gum extracted from Cyamopsis tetragonolobus, is a commercially used galactomannan where mannose/glucose ratio is 1:5 (Silveira and Bresolin, 2011). Traditionally, guar, a leguminous plant has been cultivated as feed for livestock. Guar bean is also known as cluster bean. Guar gum possesses 5-8 times the thickener activity compared to that of starch (Carbohydrate website). Galactomannan has been found to be safe and non-sensitising for dermal uses and can be used in preparation of transdermal patches (Silveira and Bresolin, 2011). Glucomannan is a neutral, non-digestible polysaccharide 
consisting of glucose and mannose in a ratio of $1: 1.6$ joined by $\beta$ $(1 \rightarrow 4)$ linkages. It is obtained from the tubers of Amorphophallus konjac and thus is also known as konjac glucomannan (KGM) or konjac gum. Its molecular weight is higher than guar gum galactomannan (Thakur and Thakur, 2015). Presence of low proportions of acetyl groups in the molecule accounts for its high water solubility (Wang et al., 2010). Because of its high fiber content, it can effectively suppress hunger and thus shows antiobesity activity. Moreover, its calorie content is also low (Carbohydrate website). It has also been used for its antidiabetic activity and is known for its cardiovascular effects (Yao et al., 2016). It has been used in traditional Asian recipes because of its thickening and gelling properties and is also regarded safe for consumption (Wang et al., 2010). KGM has been found to be toxic in male and female rats and mice after oral administration at doses $>5000$ and $>2800 \mathrm{mg} / \mathrm{kg}$ body weight, respectively with no published data on long-term toxicity or carcinogenicity (WHO Food additive series).

\section{RHEOLOGICAL PROPERTIES AND GELATION CHARACTERISTICS}

Rheological behavior of psyllium mucilage at different temperatures was found to depend on aqueous solubility of different fractions and extractability in alkali, because of presence of uronic acid in different proportions. Higher percentage of uronic acid imparted low flow index and high consistency index to gel fractions (Hojjatoleslamy et al., 2013). Psyllium mucilage exhibited shear-thinning or pseudoplastic behavior with contradictory reports on manifestation of thixotropy. Gelation is induced by interpenetrating network and cross-linking of extended chains. A characteristic spur value in the rheogram was observed with $10 \%$ mucilage owing to breakdown of internal structure. A rigid structure is formed at higher percentages of the mucilage due to formation of hydrogen bonds between water and polysaccharide molecules (Rao et al., 2011; El Sheikh 2014). Temperature increase from 20 to $50^{\circ} \mathrm{C}$, increase in salt concentration up to $0.15 \mathrm{M}$ and increase in $\mathrm{pH}$ from 2 to $10 \mathrm{did}$ not produce significant changes in the viscosity of the mucilage. At high $\mathrm{pH}(\mathrm{pH}>10)$, pores of small size, formed due to ionization of surface carboxyl groups, could be seen distributed in microstructure of psyllium gel. Since low $\mathrm{pH}$ values did not favor ionization of the carboxylic groups, mucilage viscosity was unaffected. Presence of short branches of arabinose in the alkali extractable gel fraction of psyllium rendered "weak gel "characteristics to it. (BeMiller, 2012; Farhanaky et al., 2010).

Degree of methyl esterification in pectin results in two types of pectins as HM (high methoxyl ; > 50\%) and LM pectins which subsequently affects the gel-forming ability and stabilization of the gel. LM pectins are capable of forming electrostatically stabilized gels with calcium ions with/without sucrose and are used in the fabrication of drug delivery systems. However, aqueous solutions of pectins possess lower viscosity than those of other plant-derived gums (Morris et al., 2010). Since, arabinoxylan gels exhibit high degree of $\mathrm{pH}$-independent, temperature-independent swelling in aqueous media, they can be exploited as the matrix for drug incorporation (Nino-Medina et al., 2010). Arabinoxylan gel is devoid of any characteristic taste or odor, possesses high water retention capacity and shows broad tolerance to changes in $\mathrm{pH}$ or ionic strength (Berlanga-Reyes et al., 2014; Hernandez-Espinoza et al., 2012). Rheological and structural properties of arabinoxylan gels derived from wheat bran can be changed through alteration of molecular weight, arabinoseto-xylose $(\mathrm{A} / \mathrm{X})$ ratio, ferulic acid content and polysaccharide concentrations. Rheological study was carried out on arabinoxylans extracted from maize waste water produced under two different conditions of nixtamalisation. Laccase exposure caused the polysaccharides to gel. Shorter duration of cooking and alkaline hydrolysis produced gels of fragmented morphology and heterogeneous surface (Paz-Samaniego et al., 2015). Covalent linkages present in arabinoxylan gel account for its thermal stability. The gels do not exhibit syneresis after storage for long period. Microstructural study of the arabinoxylan gels showed them to be composed of a mixture of sheets and fine strings. Therefore, diffusion paths of the entrapped molecules will be tortuous(Berlanga-Reyes et al., 2011).

Xyloglucan extracted from tamarind seed undergoes enzymatic degradation by $\beta$-galactosidase to yield thermoreversible gels at ambient temperature (Nussinovitch, 2010). Presence of side chain sugars, galactose and xylose in the native structure favor aqueous solubility and prevent gelation in aqueous medium. Hence, its aqueous solution exhibits Newtonian characteristics which remains unaffected by high or low $\mathrm{pH}$ (Bach, 2014; Jose, 2012). Gelation of xyloglucan is induced by alcohol or high concentration of sugar. Xylogucan isolated from $H$. courbaril has demonstrated Newtoninan flow at $\leq 0.5 \% \mathrm{w} / \mathrm{v}$ concentration in aqueous solution and pseudoplastic behavior at $>$ 1\% (Arruda et al., 2015). Apparent viscosity and pseudoplasticity of rice and corn starch solutions have increased by mixing with unmodified tamarind xyloglucan solution. However, carboxymethylated and hydroxypropylated xyloglucan failed to alter starch solution viscosity. High molar mass and worm-like configuration of the hemicelluloses component of xyloglucan have been held responsible for synergistic interaction with amyloses of rice and corn starches (de Freitas et al., 2015).

On dispersion in hot or cold water, galactomannans present in guar gum are hydrated immediately to produce solutions of very high viscosity at considerably low concentrations and assume flexible coil conformation. Polymeric chain entanglements are attributed to the formation of hydrogen bonds (Coviello et al., 2013). Factors affecting viscosity of dispersion are concentration, temperature, $\mathrm{pH}$ and presence of impurities. At concentration $>3 \%$, jelly-like pastes are formed which cannot be defined structurally as gels. Guar gum dispersions exhibit thixotropy with low yield value (Chudzikowski, 1971).

KGM is deacetylated and can readily form a thermally irreversible gel in presence of alkaline coagulant (WHO Food additive series). At concentrations below $0.55 \%$, KGM solution 
behaves like a Newtonian fluid which changes to pseudoplasticity at higher concentration. Increase in temperature reduces the viscosity (Chao et al., 2012).

\section{BLENDING AND CHEMICAL MODIFICATION OF NON- STARCH POLYSACCHARIDES}

Polymer-polymer blending is based on principle of physical bonding to achieve polymers of modified physical characteristics. Chemical modification such as grafting of monomers or polymers on polysaccharides by suitable crosslinking agents is necessary to improve and alter physicochemical and pharmaceutical characteristics such as hydrophilicity, aqueous solubility, solution stability, rheological properties, stimuliresponsiveness, swellability, degree of gelation, mucoadhesiveness, drug entrapment efficiency, targeting, filmforming ability and drug release kinetics (Ogaji et al., 2012; Ngwuluka et al., 2014).

Blend of psyllium with sodium alginate has been investigated for encapsulation of probiotic, Lactobacillus plantarum 15HN, Enterococcus durans 39C, with successful reports on entrapment efficiency, maintenance of cell viability, 12 $\mathrm{h}$ release in colonic medium (Haghshenas et al., $2015 \mathrm{a}, \mathrm{b}$ ). Grafting of psyllium with polymethacrylic acid yielded $\mathrm{pH}$ sensitive, biodegradable hydrogels from where swelling-induced drug release occurred preferentially in acidic medium, compared to neutral and alkaline medium. There are also reports of chemical grafting of psyllium with $\mathrm{N}$-hydroxymethylacrylamide, mixtures of acrylamide and 2-acrylamido-2-methylpropane sulphonic acid and succinic acid treatment to produce modified psyllium exhibiting altered $\mathrm{pH}$-sensitivity, swellability, biodegradability and ultimately drug release mechanism (Kumar and Sharma, 2013). Microwave irradiation has also been exploited as an efficient and rapid technique of grafting acrylamide and $\mathrm{N}$-vinyl-2-pyrrolidone onto psyllium backbone under different reaction conditions (Jatav and Agrawal, 2015; Agarwal, 2015). Acrylic acid-grafted copolymers of psyllium mucilage have been synthesized in aqueous medium with ceric ion/nitric acid redox initiator in presence of light (Kumar and Kumar, 2014). Thiol modification of psyllium husk mucilage by covalent attachment of thioglycolic acid has altered the surface morphology of psyllium with slight enhancement in crystallinity and swelling and also produced a gel with higher mucoadhesive strength. Modified gel also showed sustained drug release without any change in viscosity (Bhatia and Ahuja, 2013).

Since pectins undergo swelling in physiological fluids and may cause initial burst release of drug, they are usually combined with hydroxypropylmethyl cellulose (HPMC) to prevent the burst phenomenon (Morris et al., 2010). Colon-specific matrix tablets of ropivacine hydrochloride were produced from combination of ethylcellulose with amidated pectin and calcium salt of pectin which improved encapsulation efficiency, altered compressibility and drug release profile (Li Fang et al., 2008). Degree of gelation and solubility of pectin are influenced by molecular weight of the pectin, degree and nature of substitution such as amidation, esterification, methoxylation, thiolation, quaternisation, sulfation, oxidation and also salt formation (Chen et al., 2015; Pant et al., 2015; Morris et al., 2010). Grafting of vinyl acetate on pectin resulted in polymer of higher crystallinity and better thermal stability in comparison to native pectin (Mishra et al., 2016). Enzymatic grafting of ferulic acid oxidation products onto pectin modified numerous inherent characteristics such as morphological surface, viscosity, hygroscopicity, thermal stability and biological activity (Karaki et al., 2016).

Arabinoxylan has been added at low ratio to agar or cassava starch to form films of different mechanical strength and water vapor permeability (WVP). Although, AX-starch formed homogeneous blend, it formed a heterogeneous blend with agar. WVP was reduced with $\mathrm{AX}$ but mechanical strength did not improve (Mikkonen, 2012). The structural properties of ferulated arabinoxylan (FAX) obtained from maize bran such as ferulic acid content, degree of substitution, chemical structure, molecular weight distribution depend on the processing conditions of the nixtamalisation. These, in turn affect the rheological parameters, gelling ability and functional characteristics of the polysaccharides. The gels produced were found to possess macroporous and heterogeneous structure (Martinez-Lopez et al., 2013). Carboxymethylated psyllium arabinoxylan has been found to possess higher crystallinity, better thermal stability and lower viscosity (Bhatia and Ahuja, 2015).

Xyloglucan has been effectively combined with sodium alginate in the development of sustained release, temperaturesensitive gel for oral administration to geriatric patients in treatment of dysphagia and also sustained release beads with metformin hydrochloride. Inclusion of alginate resulted in slow gel erosion and the formulation exhibited diffusion-controlled drug release (Itoh et al., 2010). The beads exhibited pH-dependent, zero-order drug release. Super case II transport is the driving mechanism for drug release (Nayak et al., 2015). Mechanical properties, hydrophobicity, crystallinity, sorption ability, swelling behavior and drug release characteristics were favourably modified by blending xyloglucan with chitosan (Simi and Abraham, 2010). Thermoreversible gelation can be induced and gels of varying stiffness can be produced by blending of tamarind seed xyloglucan with different gallate analogs. Relative position of acetoxyl and hydroxyl groups influenced the gelling potency (Yan et al., 2016). Enzymatic oxidation of terminal galactose residues by galactosidase followed by chemical derivatisation have been employed to produce carboxylated, sulphated, and alkylaminated derivatives of xyloglucans. Glass transition temperature of xyloglucan is lowered by regioselective chemical modification of side chains through periodate oxidation and subsequent reduction steps (Jose, 2012). Carboxymethyl xyloglucan (CMXG) possesses higher stability, viscosity and swelling index compared to the native form. Matrix tablets produced from blend of CMXG and HPMC K 100M showed controlled drug release for 8-10 hrs. Initial burst phase was delayed by addition of HPMC whereas CMXG exerted retardant effect on the later phase of drug release 
profile (Madgulkar et al., 2013). Thiolated xyloglucan has been reported to possess better mucoadhesive properties in comparison to the parent polysaccharide and exhibits enhanced drug permeation profile (Mahajan et al., 2013). Ring-opening polymerization has been employed for grafting of L-lactide monomer on xyloglucan leading to polymer with lower solubility, reduced moisture uptake and improved adsorption onto cellulose fibres (Marais et al., 2012). Polymeric, chemical cross-linking of xyloglucan with chitosan has been done where aldehydric groups of polysaccharide formed Schiff bases with primary amino groups of chitosan (Nygaard et al., 2015). Grafting of polymethyl methacrylate (PMMA) / acrylonitrile with xyloglucan has been successfully carried out employing ceric ammonium nitrate as initiator. The resulting product showed improved shelf-life and thermal stability without any effect on its hydrophilicity (Mishra and Malhotra, 2012). Increased infiltration levels for astrocytes and neurites has been observed with higher concentrations of xyloglucan-graft-poly-D-lysine hydrogels in comparison to thermally gelling xyloglucan hydrogels and thus can be developed as scaffolds in neural tissue engineering (Nisbet et al., 2010).

Polymeric blending of guar gum and poly(vinyl alcohol) has been used successfully in formation of monodisperse, highly stable silver nanoparticles, possessing antibacterial and antioxidant activities (Das et al., 2015). Cross-linking of guar galactomannan with glutaraldehyde actually enhanced release of theophylline from tablet hydrogel in comparison to tablet using un-cross-linked galactomannan. This was attributed to decreased resistance to diffusing drug molecules. Carboxymethyl guar gum has been used in formation of terbutaline sulfate transdermal films of sufficient mechanical strength and stability and exhibiting zero-order release. Polyacrylamide-grafted guar gum has been employed to develop pH-sensitive hydrogel (Silveira and Bresolin, 2011). Phosphorylated derivative of guar gum galactomannan with varying degree of substitution was obtained via cross-linking of polysaccharide chains with $\mathrm{POCl}_{3}$. Degree of substitution affected the polymer conformation in aqueous solution, stiffness of gel and free radical scavenging activity (Wang et al., 2014).

Blending of KGM/KGM hydrolysates with chitosan, xanthan gum or poly(acrylic) acid has been done in various studies to produce films of desirable physicochemical and mechanical properties (Li et al., 2009; Xiao et al., 2000; Li et al., 2006, Yu et al., 2006). Addition of KGM to binary mixtures of kappacarrageenan and iota-carrageenan resulted in an initial increase in the elastic modulus till a certain stoichiometric ratio, beyond which a plateau was observed. Further increase in concentration of KGM increased fracture strain of the mixture (Brenner et al., 2015). Similar observations were made with kappa-carrageenanKGM binary gels where stoichiometric ratio of the components played a crucial role. No such observation was made when locust bean gum was added to the mixture (Brenner, 2013). KGM has been found to modify the sensory hardness and stickiness of gelatin gels and subsequently alter mastication and swallowing of the gels. Elastic modulus was increased and there was a slight reduction in fracture stress. Melting temperature was maintained below body temperature (Tomczyńska-Mleko et al., 2014). KGM decreased the water solubility index, water absorption capacity, gelatinization temperature of wheat starch/potato starch. Swelling power of the starch granules was unaffected. Viscosity of starch paste was increased by KGM (Varela et al., 2016). Attempts for chemical modification of konjac glucomannan have been made through palmitoylation, grafting, carboxymethylation, sulfation and quaternisation to yield bio-functional materials. Carboxymethylation of KGM led to decrease in water absorption, adsorption and solubility and resultant increase in hydrophobicity. Equilibrium moisture content of carboxymethylated KGM is lower than that of $\mathrm{KGM}$ when determined in the relative humidities of $30-95 \%$ at $25^{\circ} \mathrm{C}$ (Xiao et al., 2015). Carboxymethyl KGM grafted monomethoxy PEG copolymer with grafted PEG oligomers has demonstrated improved aqueous solubility and lower viscosity at low shear frequency in comparison to non-PEGylated KGM (Xia et al., 2010). There have been different studies on various aspects of sulfated konjac glucomannan. There was marked increase in the viscosity of sulfated derivative-xanthan gum complex, probably due to hydrogen bond interaction (Lu Yan et al., 2016). Modified KGM has demonstrated potent anti-HIV activity as well as anticoagulant activity. Biological activity of sulfated KGM might have occurred due to electrostatic interaction between sulfate and amino groups (Huang et al., 2015; Bo et al., 2013). Radiationinduced graft copolymerization of KGM with acrylic acid has resulted in superabsorbent polymer with a porous microstructure. Diffusion of water followed anomalous diffusion model. Multivalent cations, increase in temperature, shift in $\mathrm{pH}$ lowered water absorption capacity (Wu et al., 2013). It has shown potential to be used as novel adsorbent of blood low density lipoprotein and very low density lipoprotein cholesterol (Zhang et al., 2008).

\section{APPLICATIONS OF NON-STARCH POLYSACCHARIDES IN DESIGN OF DRUG DELIVERY SYSTEMS}

Since they are capable of fulfilling specialized functions in ensuring better drug delivery, attempts are being made to harness the full potential of non-starch polysaccharides obtained from plants in developing dosage forms with tailored and sitespecific drug release. Their use as tablet superdisintegrant and application in the fabrication of hydrogels, multiparticulates, gastroretentive dosage forms, matrix tablets, nasal drug delivery, mucoadhesive drug delivery system and colon-specific systems will be discussed in the forthcoming sections.

\section{HYDROGELS}

Thermoreversible phase transformation resulted in formation of xyloglucan hydrogels when gallic acid was added to it. Entrapped gallic acid could be released by non-Fickian diffusion and such hydrogels can be used for topical routes for sustained drug delivery (Hirun et al., 2016). In situ, thermoreversible and mucoadhesive gel of lidocaine hydrochloride has been developed from xyloglucan by use of Lutrol F127 for use in treatment of periodotosis (Pandit et al., 2016). Lower critical 
solution temperature of modified xyloglucan hydrogels can be achieved by removal of galactose residues, which also enhances the hydrophobicity of the polymer (Gandhi et al., 2015). Stimuliresponsive xyloglucan hydrogels are sensitive to temperature changes and undergo sol-gel transformation for temperature rise from $25^{\circ} \mathrm{C}$ to $37^{\circ} \mathrm{C}$. Increase in temperature causes polymer chain degradation and exposure of hydrophobic domains (Kushwaha et al., 2012). Polymer-ion complexes between guar gum and borate results in the formation of three-dimensional network. During swelling of hydrogel, rearrangement of intermolecular chains and anisotropic elongation occurs. Drug diffusion from the hydrogel is affected by network architecture and viscous drag exhibited by quite large number of elastically inactive chains that can fluctuate among the meshes. Such weak van der Waals interactions can hinder drug release when mesh size is higher than size of drug molecule (Coviello et al., 2013). Guar gum-alginate conjugate cross-linked with glutaraldehyde formed a hydrogel from which bovine serum albumin released slowly at $\mathrm{pH} 1.2$ in contrast to that at pH 7.4 (Thakur and Thakur, 2015). Photopolymerised guar gum-methacrylate hydrogel was able to entrap human endothelial cell line experimentally which lost viability at higher macromolecular concentrations (Iqbal and Hussain, 2010). Hydrogels formed by covalent cross-linking between waterextractable arabinoxylan and ferulic acid have low entrapment efficiency. To overcome this disadvantage, arabinoxylan aerogels have been prepared with supercritical carbondioxide. Aerogels thus formed presented heterogeneous, porous organization, capable of undergoing rehydration by non-Fickian diffusion (Marquez-Escalante et al., 2013). Semi-interpenetrating network hydrogel of KGM and poly(aspartic acid) have been prepared using trisodium trimetaphosphate as cross-linking agent( $\mathrm{Lu}$ et al., 2015).

\section{MULTI-PARTICULATE DRUG DELIVERY SYSTEM (NANOPARTICLES AND MICROSPHERES)}

Polyelectrolyte complex nanoparticles of ibuprofen have been prepared from carboxymethylated psyllium arabinoxylan and chitosan. Nanoparticles possessed low polydispersity and drug release followed Higuchi kinetics with release up to $10 \mathrm{hrs}$ (Bhatia and Ahuja, 2015). Nanoparticles of cross-linked guar gum increased uptake of tamoxifen citrate by mammillary tissue of albino female mice (Thakur and Thakur, 2015). Microspheres formed from maize bran arabinoxylan showed heterogeneous structure. They have been found to contain macro-cavities with honey-comb like structure and possess mesoporous organization. Factors affecting microsphere microstructure are content and distribution of ferulic acid covalent cross-linking structures forming the gel network (Martinez-Lopez et al., 2013). Metronidazole has been encapsulated into microspheres of arabinoxylan gelled with calcium chloride, produced by spray drying technique. Ionotropic gelation method retarded drug release in acidic medium (Lee et al., 2015). Xyloglucan microspheres have been investigated for their use as dry powder inhalers for pulmonary delivery of montelukast in animal model (Mahajan and Gundare, 2013). Release of indomethacin from xyloglucan beads was affected by drug content and $\mathrm{pH}$ of the medium. Enteric coating of xyloglucan beads with specific grades of Eudragit could achieve colon-specific release of encapsulated drug (Nussinovitch et al., 2010). Hydrogel microspheres have been developed from graft copolymer of guar gum with acrylamide by cross-linking with glutaraldehyde. Release of entrapped verapamil and nifedipine occurred by non-Fickian diffusion (Thakur and Thakur, 2015).

\section{GASTRORETENTIVE DOSAGE FORMS}

Non-effervescent, expandable gastro-retentive dosage form has been successfully designed by mixing plant mucilloid, psyllium husk with HPMC K4M and HPMC K 15M, for a model water-soluble drug, Carvedilol phosphate. Drug release occurred from the resultant hydrogel network via diffusion/relaxation controlled process (Majee et al., 2014). Pectin-HPMC and crosslinked low methoxylated pectin-sodium alginate hollow beads were used to sustain release of gliclazide in gastric fluid where they remained buoyant for $12 \mathrm{hrs}$. Swelling-induced drug release occurred through Fickian diffusion mechanism(Awasthi and Kulkarni, 2014; Talukder and Fassihi, 2004).

\section{MATRIX TABLETS}

Extended release matrix tablets of diclofenac sodium prepared with high percentages of Isapgula husk exhibited structural integrity, initial zero-order drug release followed by first-order kinetics (Raja et al., 2014). With proper selection of fillers, the physico-mechanical properties of psyllium can retard release of water-soluble drug from tablets (Rafie et al., 2012). Matrix tablets of diltiazem hydrochloride have been fabricated from blend of xyloglucan-gellan gum-sodium carboxymethylcellulose (Thakur and Thakur, 2015). High degree of swelling, viscosity building effect and gel-forming ability of guar gum during tablet disintegration and dissolution, can lead to delayed disintegration and regulated drug release (Silveira and Bresolin, 2011).

Guar gum-based controlled release matrix tablets of metoclopramide hydrochloride, diclofenac sodium, metformin hydrochloride, theophylline have been formulated and effect of fillers like lactose monohydrate, dicalcium phosphate, microcrystalline cellulose have been investigated (Thakur and Thakur, 2015).

\section{AS TABLET SUPERDISINTEGRANT}

Psyllium mucilage can act as a tablet disintegrant owing to high swelling index and its ability to form numerous water channels. It has been reported to be a better disintegrant than maize starch in the famotidine tablets prepared by direct compression, when added at a concentration of $10 \% \mathrm{w} / \mathrm{w}$. However, at higher concentration of the polysaccharide, the 
process of disintegration was hindered due to the binding property of mucilage (Mehta et al., 2011). In another study, amlodipine besylate tablets disintegrated in 11.69 secs, when psyllium mucilage was used and hence it is considered as a natural superdisintegrant (Alam et al., 2014). Fast disintegrating tablets of glipizide have been developed with guar gum (Thakur and Thakur, 2015).

\section{NASAL DRUG DELIVERY}

Pectin-based intranasal gel or pectin-chitosan nasal inserts have been exploited as a means of delivering therapeutically safe levels of opioid analgesic and improved bioavailability (Watts and Smith, 2009). The percentage of pectin in the insert exerted significant influence on water permeability, mucoadhesion and drug diffusion (Luppi et al., 2010). Nasal insert formulation composed of xanthan gum and guar gum (1:5) effectively enhanced residence time and release of metoclopramide hydrochloride in the nasal cavity and exhibited good bioadhesion. Freeze-dried formulation showed higher viscosity than either of the gums alone (Dehghan and Girase, 2010).

\section{MUCOADHESIVE DRUG DELIVERY}

Psyllium mucilage has been used along with Carbopol 974P in fabrication of controlled release buccoadhesive tablets of propranolol hydrochloride. Excellent bioadhesion was attributed to the formation of hydrogen bonds between the hydrophilic groups of the mucilage and the mucus layer. Drug release followed Higuchi, zero-order kinetics or Peppas model, depending on the concentration of the mucilage in the formulation (Saeedi et al., 2014). Molecular weight and chain flexibility govern the mucoadhesive properties of the pectins (Morris et al., 2010). Primary amine groups in pectin derivatives usually enhance their adhesion property to various mucosal surfaces (Pant et al., 2015). Novel xyloglucan-block-poly( $\epsilon$-caprolactone)nanoparticles coated with chitosan improved their mucoadhesive property due to the presence of strong electrostatic interactions with mucin (Mazzarino et al., 2014).

Cytotoxicity, genotoxicity, erythrocyte toxicity and subacute toxicity were studied for uncoated block copolymer nanoparticles. There was no detectable reduction in viability of MRC-5 human fetal lung fibroblast cells and no DNA damage was reported. No clinical signs of toxicity, mortality, weight loss, or haematological and biochemical alterations could be observed in $\mathrm{BALB} / \mathrm{c}$ mice after treatment with nanoparticles at a dose of 10 mg.kg body weight, either orally or intraperitoneally (Mazzarino et al., 2015). Tamarind seed polysaccharide has been employed in preparation of mucoadhesive buccal films for water soluble drug, rizatriptan benzoate (Avachat et al., 2013).

Mucoadhesive xyloglucan microspheres have been prepared for delivery of glipizide which have been shown to exert hypoglycemic effect in vivo (Mahajan and Gundare, 2013).

\section{COLON-SPECIFIC DRUG DELIVERY}

Linear polysaccharides of non-starch type are protected against enzymatic proteolytic action of the gastric or intestinal juice and remain intact till they reach the colon where they are degraded by resident microbial flora. Moreover, different derivatisation approaches and cross-linking reactions modify their aqueous solubility, gelation property, mucoadhesion tendency, tablet disintegration, biodegradation and finally drug release profile. These modified non-starch polysaccharides are free from dose-dumping phenomenon and seem suitable for controlled drug release and site-specific delivery to the colonic region (HernandezEspinoza, 2012; Thakur and Thakur, 2015). Pectin-zein hydrogel beads can be exploited as colon-specific drug delivery system because of their desirable swelling and porosity characteristics and their ability to resist protease attack (Pant et al., 2015). Timedependent and microflora-triggered pulsatile capsule of 5aminosalicylic acid has been designed by using two types of polymer combination for plug tablet-HM pectin/lactose and LMpectin/HPMC to ensure suitable lag time and ultimately colon specificity (Liu et al., 2012). Colon-targeted release of budenoside was obtained from pellets coated with different ratios of pectin and different grades of Eudragits or Surelease. Drug release was governed by nature of adjuvant polymer, ratio of polymers in the film and thickness of the film (Varshosaz et al., 2012). Filmcoated pellets of pectin-ethylcellulose showed varying degree of hydration and swelling induced by bacterial polysaccharidases and thus found suitable for development into colon-specific dosage form. In vitro-in vivo correlation could be established successfully from pharmacokinetic studies in dogs (Li-Fang et al., 2008). Water-extractable arabinoxylan (WEAX) gels are known for their porous nature, high water absorption capacity and their susceptibility to bacterial degradation by intestinal microflora. Thus, they can be specifically employed in development of colonspecific dosage forms (Berlanga-Reyes et al., 2014). Probiotic Bifidobacterium longum has been successfully entrapped in WEAX gels resulting in imperfect honeycomb structure with slight loss in gel elasticity (Morales-Ortega et al., 2014). Guar gum has been investigated as a carrier for colon-specific delivery of 5-fluorouracil and mebendazole in human volunteers (Krishnaiah et al., 2003a,b). Cross-linked guar gum with little amounts of titanium yielded colon-specific tablets from where the entrapped fluorescent probe, fluorescein isothiocyanate released slowly in colonic fluid during in vitro studies (Silveira and Bresolin, 2011). Polyelectrolyte hydrogel synthesized from cationic guar gum and anionic polyacrylic acid demonstrated nonFickian drug diffusion in $\mathrm{pH} 7.4$ preferentially, thereby enabling its use in colon-specific drug delivery (Thakur and Thakur, 2015). Konjac glucomannan, copolymerized with acrylic acid and crosslinked by bis(methacryloylamino)-azobenzene or N,N-methylenebis(acrylamide) formed a hydrogel which showed $\mathrm{pH}$-dependent release of entrapped drug molecules. Release was comparatively slow in $\mathrm{pH} 2.2$ than in $\mathrm{pH} 7.4$ and continued for 10 days obeying zero-order kinetics. Since, swelling of hydrogel, degradation and 
drug release was higher in colonic $\mathrm{pH}$, grafted and cross-linked KGM can be used for development of colon-specific dosage forms (Thakur and Thakur, 2015). Colon-specific pulsatile delivery system has been fabricated by enclosing rapidly-disintegrating tablet of 5-aminosalicylic acid within an impermeable capsule body sealed with KGM-HPMC-lactose plug. Biphasic drug release was characterized by initial lag time followed by a rapid release phase. Factors affecting lag time were KGM-HPMC-lactose ratio, type of HPMC and plug weight. Data from bioavailability studies revealed drug in plasma 5 hrs after oral administration (Liu et al., 2012).

\section{SWELLING BEHAVIOR AND DRUG RELEASE KINETICS FROM NON-STARCH POLYSACCHARIDE BASED DOSAGE FORMS}

Swelling kinetics and release dynamics of the psylliumacrylic acid hydrogel has been studied extensively and it has been found to be a promising vehicle for delivery of proteins, amino acids and antibiotics. Drug release is controlled by two simultaneous processes of polymer chain relaxation by influx of water or phosphate buffer ( $\mathrm{pH}$ 7.4) and drug diffusion out of the swollen matrix (Singh et al., 2007;Singh, 2007). Being high in fiber content, psyllium forms gel rapidly in the presence of gastrointestinal contents and hence delays drug absorption (Singh, 2007).

Formation of physical non-covalent gel network of pectin is controlled by solubility, composition of the hydrophilic matrix and the rate of influx of the dissolution medium, Diffusioncontrolled drug release follows (Li-Fang et al., 2008).

Swelling ratios at equilibrium swelling of arabinoxylan gels, extracted from wheat bran and maize brain have found to be different. Lower value obtained with wheat bran can be explained by formation of a highly compact network structure which limits water absorption (Berlanga-Reyes et al., 2014). Model proteins like insulin, ovalbumin and bovine serum albumin were entrapped and homogeneously distributed in wheat bran arabinoxylan gels at low mass ratio or protein/polysaccharide ratio. Under experimental conditions of the study, low amount of entrapped proteins diffused out of the gels which indicates that protein release can be completed only after polysaccharide degradation by colonic microflora. For insulin, diffusion coefficient was dependent on mass ratio. Diffusion of entrapped proteins depends on molecular weight and followed Higuchi kinetics where cumulative percent release is a function of square root of time. It is to be noted that protein entrapment did not affect covalent cross-linking in the mixed gels (Berlanga-Reyes et al., 2014; Berlanga-Reyes et al., 2009). It has also been reported that lycopene oxidation can be prevented and its release can be monitored by delivering it through arabinoxylan gels (Hernandez-Espinoza et al., 2012). Swelling of arabinoxylan microspheres occurred due to penetration of water by Fickian diffusion. Rate of water influx is lower than polymer chain relaxation rate (Martinez-Lopez et al., 2013). CMXG revealed swelling index to depend on time and concentration of CMXG.
This happens because the polymer takes time to swell in presence of water. High swelling index prolongs time taken for water molecules to move inwards into tablet core and produces sustained release profile. Anomalous transport mechanism was followed during release of tramadol from matrix tablets (Madgulkar et al., 2013). Tamarind seed polysaccharide based hydrophilic matrix tablets showed different release kinetics with different drugs. Drug release mechanism was found to depend on aqueous solubility of drug, type of diluents used in the formulation. Anomalous transport was the principal mechanism for release of soluble drugs. Insoluble drugs followed case II transport or zero-order release kinetics and controlled by the processes of diffusion and erosion. Slow drug release is attributed to the presence of hydrogen bonds and formation of gel barrier (Sumathi and Roy, 2002).

In case of guar gum, swelling of matrix occurs sequentially. Firstly, there is hydration and swelling of galactomannan chains, followed by gelation. Solvent influx leads to entrapped drug molecules within the core from where dissolved drug is released by disentanglement or relaxation of polymer chains and subsequent erosion of the matrix. Polyacrylamidegrafted guar gum undergoes $\mathrm{pH}$-dependent swelling to release water-soluble drugs in a non-Fickian manner (Silveira and Bresolin, 2011).

Study of thermodynamic behavior during swelling of native KGM revealed it to be a three-step process where self-association of hydroxyl groups played a crucial role in swelling via its effect on surface tension. Native KGM is found to consist of lamella structure units comprising both granular crystalline and amorphous regions. Water adsorption, absorption and solubility of KGM can be reduced by carboxymethylation. Modified water binding properties can be employed in pharmaceutical field. Equilibrium moisture content of the chemically modified KGM is lower than its native counterpart at relative humidities of $30-95 \%$ at $25^{\circ} \mathrm{C}$ (Xiao et al., 2015). Swelling and drug release from novel polyelectrolyte beads of carboxymethylated KGM-chitosan were found to be $\mathrm{pH}$-sensitive and very suitable for delivery of protein and peptide drugs (Du et al., 2006). Study of drug release mechanism from KGM-cassava starch blend showed that release of theophylline from the films obeyed Higuchi's kinetic model. Combination of diffusion and polymer relaxation contributed to anomalous transport of drug following non-Fickian kinetics (Nair et al., 2011).

\section{CONCLUSION}

Non-starch polysaccharides isolated from plants are found to possess immense potential to be employed in the fabrication of drug delivery systems to achieve tailored and/or sitespecific drug release. Most of them are used as food additives and are generally recognized as safe for human consumption. In their native, grafted, chemically or enzymatically modified forms as well as in physical blends, they have demonstrated excellent rheological properties and swelling behavior which can be utilized for slow drug diffusion out of matrix devices. In near future, there 
will be a shift in global trend towards use of these eco-friendly, non-starch polysaccharides derived from plants in pharmaceutical field extensively.

\section{Financial support and sponsorship: Nil.}

Conflict of Interests: There are no conflicts of interest.

\section{REFERENCES}

Agarwal N. Synthesis and characterization of psyllium graft copolymer of N-vinyl 2-pyrolidone with microwave irradiation. J. Chem. Chem. Sci. 2015; $5: 325-32$.

Arruda IRS, Albuquerque PBS, Santos GRC, Silva AG, et al. Structure and rheological properties of a xyloglucan extracted from Hymenaea courbaril var. courbaril seeds. Int J. Biol. Macromol. 2015; 73 : 31-8.

Avachat AM, Gujar KN, Wagh KV. Development and evaluation of tamarind seed xyloglucan-based mucoadhesivebuccal films of rizatriptan benzoate. Carbo Polym. 2013; 91 : 537-542.

Awasthi R, Kulkarni GT. Development of novel gastroretentive drug delivery system of gliclazide: hollow beads. Drug Dev Ind Pharm. 2014; 40 :398-408.

Bach J. Determination of the GRAS status of the addition of tamarind seed polysaccharide to conventional foods as a stabilizer and thickener. 2014; $\quad$ GRAS (GRN)No.503.http://www.fda.gov/Food/IngredientsPackagingLabeling/G RAS/NoticeInventory/default.htm (accessed on 27.11.2015).

BeMiller JN. Quince seed, Psyllium seed, Flax seed and Okra gums in Industrial Gums : Polysaccharides and their derivatives. Whistler R(Ed.). Elsevier-Technology and Engineering. (2012).

Berlanga-Reyes CM, Carvajal-Millán E, Lizardi-Mendoza J, Rascón-Chu A, Marquez-Escalante JA, Martínez-López AL. Maize arabinoxylan gels as protein delivery matrices. Molecules. 2009; 14 : 1475-82.

Berlanga-Reyes CM, Carvajal-Millan E, Hicks KB, Yadav MP, Rascón-Chu A, et al. Protein/arabinoxylans gels: effect of mass ratio on the rheological, microstructural and diffusional characteristics. Int $\mathrm{J}$ Mol Sci. 2014; $15: 19106-18$.

Berlanga-Reyes CM, Carvajal-Millan E, Lizardi-Mendoza J, Islas-Rubio AR, Rascón-Chu A. Enzymatic cross-linking of alkali extracted arabinoxylans: gel rheological and structural characteristics. Int J Mol Sci. 2011; 12: 5853-61.

Bhatia M, Ahuja M. Thiol modification of psyllium husk mucilage and evaluation of its mucoadhesive applications. The Scient. World J. 2013; Article ID 284182, 7 pages.

Bhatia M, Ahuja M. Psyllium arabinoxylan: carboxymethylation, characterization and evaluation for nanoparticulate drug delivery. Int J Biol Macromol. 2015; 72 : 495-501.

Bindu D, Bharath P, Kumar CA. Psyllium : a potential carrier to control drug delivery. Int Res J Pharm. 2012; $3: 39-45$.

Biswas Majee S, Karmakar D, Ghosh N, Roy Biswas G. Fabrication and characterisation of plant mucilloid-based non-effervescent expandable gastroretentive drug delivery system. Lat Am J Pharm. 2014; $33: 1357-1362$.

Bo S, Muschin T, Kanamoto T, Nakashima H, Yoshida T. Sulfation and biological activities of konjac glucomannan. Carbohydr Polym. 2013; 94 :899-903.

Brenner T, Tuvikene R, Fang Y, Matsukawa S, Nishinari K . Rheology of highly elastic iota-carrageenan/kappa-carrageenan/xanthan/ konjac glucomannan gels. Food Hydrocoll. 2015; 44 : 136-44.

Brenner T, Wang Z, Achayuthakan P, Nakajima T, Nishinari K. Rheology and synergy of $\kappa$-carrageenan/locust bean gum/konjac glucomannan gels. Carbohydr Polym. 2013 ; 98 :754-60.

Carbohydrate website. Available at: http://www.scientificpsychic.com/fitness/carbohydrates2.html [Accessed on November 25, 2015].
CarePsyllium website. Available at: http:// www.caremoligroup.com/sites/ default/files/ CarePsyllium.pdf [Accessed on November 29, 2015].

Chao W, Mei X, Wen-ping LV, Pei Q, Yuan-yuan G, Dongsheng L. Study on rheological behavior of konjac glucomannan. Physics Procedia. International Conference on Medical Physics and Biomedical Engineering . 2012; $33: 25-39$

Chen $\mathrm{M}$, Zhou Y, Huang J, Zhu P, Peng X, Wang Y. Liposome-based delivery systems in plant polysaccharides. J Nanomat. 2012; Article ID 682545, 4 pages.

Chudzikowski RJ. Guar gum and its applications. J Soc. Cosmet. Chem. 1971; $22: 43-60$.

Das T, Yeasmin S, Khatua S, Acharya K, Bandyopadhyay A. Influence of a blend of guar gum and poly(vinyl alcohol) on long term stability, and antibacterial and antioxidant efficacies of silver nanoparticles. RSC Adv. 2015; 5 : 54059-69.

de Freitas RA, Woehl MA, Kaminski GAT, da Silva R, Sierakowski MR. Rheological description of the interaction of xyloglucan and starches: effect of the amylose content in starches. CyTA - J. Food. $2015 ; 13: 235-42$.

Dehghan MH, Girase M. Freeze-dried xanthan/guar gum nasal inserts for the delivery of metoclopramide hydrochloride. Iran. J. Pharm. Res. 2012; $11: 513-21$.

Du J, Dai J, Liu JL, Dankovich T. Novel pH-sensitive polyelectrolyte carboxymethylKonjacglucomannan-chitosan beads as drug carriers. React Funct. Polym. 2006; 66 : 1055-61.

El Sheikh DM. Rheological characteristics of Arabic gum suspension and Plantago seeds mucilage. J Amer. Sci. 2014; 10 : 18-24.

Erum A, Bashir S, Saghir S, Tulain UR, Saleem U, Nasir M, Kanwal F, Hayat Malik MN. Acute toxicity studies of a novel excipient arabinoxylan isolated from Ispaghula (Plantago ovata) husk. Drug Chem Toxicol. 2015; $38: 300-305$.

Farahnaky A, Askari H, Majzoobi M, Mesbahi Gh. The impact of concentration, temperature and $\mathrm{pH}$ on dynamic rheology of psyllium gels. J. Food Engg. 2010; $100: 294-301$.

Gandhi A, Paul A, Sen SO, Sen KK. Studies on thermoresponsive polymers: Phase behaviour, drug delivery and biomedical applications. Asian J. Pharm. Sci. 2015; 10 : 99 -107.

Haghshenas B, Nami Y, Haghshenas M , Barzegari A, et al. Effect of addition of inulin and fenugreek on the survival of microencapsulated Enterococcus durans 39C in alginate-psyllium polymeric blends in simulated digestive system and yogurt. Asian J. Pharm. Sci. 2015; 10 : 350-61.

Haghshenas B, Abdullah N, Nami Y, Radiah D, Rosli R, Yari Khosroushahi A. Microencapsulation of probiotic bacteria Lactobacillus plantarum $15 \mathrm{HN}$ using alginate-psyllium-fenugreek polymeric blends. J. Appl. Microbiol. 2015; 118:1048-57.

Hernández-Espinoza AB, Piñón-Muñiz MI, Rascón-Chu A, Santana-Rodríguez VM, Carvajal-Millan E (2012). Lycopene/ Arabinoxylan gels: rheological and controlled release characteristics Molecules. 17 : 2428-2436. doi:10.3390/molecules17032428.

Hirun N, Tantishaiyakul V, Sangfai T, Rugmai S, Soontaranon S. Nano-structure, phase transition and morphology of gallic acid and xyloglucan hydrogel. Polym. Bull. 2016; 73 : 2211-26.

Hojjatoleslamy M, Raisi F, Asadi M, Mohammadian M, Samanian N. Rheological properties of the gum fractions from psyllium (Plantago psyllium L.). J. Herbal Drugs 2013; 4 : 69-74, 2013.

Huang H, Wang G, Chen J, Zhou W. Characterization of sulfate groups and assessment of anti-coagulant activity of glucomannan sulfate prepared from konjac glucomannan Trop. J. Pharm. Res. 2015; 14 : 1217 24.

Hussain MA, Muhammad G, Jantan I, Syed Bukhari NA. Psyllium arabinoxylan: a versatile biomaterial for potential medicinal and pharmaceutical applications. Polym. Rev. 2016; $56: 1-30$.

Iqbal DN, Hussain EA. Physiochemical and pharmaceutical properties of guar gum derivatives. Asian J. Chem. 2010; 22 : 7446-52.

Itoh K, Tsuruya R, Shimoyama T, Watanabe H, Miyazaki S, D'Emanuele A, Attwood D. In situ gelling xyloglucan/alginate liquid 
formulation for oral sustained drug delivery to dysphagic patients. Drug Develop. Ind. Pharm. 2010; 36 : 449-55.

Jatav P, Agrawal N. Synthesis and characterization of graft copolymer of methacrylamide onto psyllium. Int. J. Latest Res. Sci. Technol. 2015; $4: 1-7$.

Jose JK. Xyloglucan-based polymers and nanocomposites modification, properties and barrier film applications. Doctoral Thesis submitted to KTH, Royal Institute of Technology, Division of Biocomposites . Stockholm. Sweden, 2012.

Karaki N, Aljawish A, Muniglia L, Humeau C, Jasnieweski J . Physicochemical characterization of pectin grafted with exogenous phenols. Food Hydrocoll. 2016; 60 : 486-93.

Krishnaiah YSR, Satyanarayana S. In vivo pharmacokinetics in human volunteers: oral administered guar gum-based colon-targeted 5fluorouracil tablets. Eur. J. Pharm. Sci. 2003b ; 19 : 355-62.

Krishnaiah YSR, Veer Raju P, Dinesh Kumar D, Satyanarayana V, Karthikeyan RS, Bhaskar P. Pharmacokinetic evaluation of guar gum-based colon-targeted drug delivery systems of mebendazole in healthy volunteers. J Contr Rel. 2003a ; 88 : 95-103.

Kumar D, Kumar S. Grafting of acrylic acid on to Plantago psyllium mucilage IOSR J. Appl. Chem. (IOSR-JAC) 2014; 7 : 76-82.

Kumar R, Sharma K. Biodegradable polymethacrylic acid grafted psyllium for controlled drug delivery systems. Front Chem Sci Eng. 2013; $7: 116-22$.

Kushwaha SKS, Saxena P, Rai AK. Stimuli sensitive hydrogels for ophthalmic drug delivery: A review. Int. J. Pharm. Invest. 2012; 2 : 54-60.

Lee CJ, Nah CS, Teng CS, Jun WW, Saravanan M. Spray dried calcium gelled arabinoxylan microspheres: A novel carrier for extended drug delivery. Chemical Papers. 2015; 69 : 1325-30.

Li B, Peng, J, Yie X, Xie,B. Enhancing physical properties and antimicrobial activity of konjac glucomannan edible films by incorporating chitosan and nisin. J Food Sci. 2006; 71: C174-C178.

Li Q, Qi W, Su R, He Z. Preparation and characterization of enzyme-modified konjacglucomannan/xanthan blend films. 2009; 20:299. 310.

Li-Fang F, Wei H, Min B, Qing D, Bai X, Yong-Zhen C, DeYing C. Biphasic drug release: permeability and swelling of pectin/ethylcellulose films, and in vitro and in vivo correlation of filmcoated pellets in dogs. Chem Pharm Bull. 2008; $56: 1118-25$.

Liu J, Zhang L, Hu W, Tian R, Teng Y, Wang C. Preparation of konjac glucomannan-based pulsatile capsule for colonic drug delivery system and its evaluation in vitro and in vivo. Carbo. Polym. 2012; 87 : 377-382.

Liu J, Zhang L, Jia Y, Hu W, et al. Preparation and evaluation of pectin-based colon-specific pulsatile capsule in vitro and in in vivo. Arch. Phar. Res. 2012; 35 : 1927-34.

Lowery M. New Pullulan-based capsules offer unique benefits for supplement manufacturers and healthy lifestyle market worldwide. Available at

http://www.nutraceuticalsnow.com/articles/2012/08/27/new-pullulanbased-capsules-offer-unique-benefits-supplement-manufacturers-healthy/ .

Lu J, Li Y, Hu D, Chen X,et al. Synthesis and properties of $\mathrm{pH}-$, thermo-, and salt-sensitive modified poly(aspartic acid)/poly(vinyl alcohol) IPN hydrogel and its drug controlled release. Hindawi Publishing Corporation BioMed Res. Int. 2015; Article ID 236745, 12 pages.

Luppi B, Bigucci F, Abruzzo A, Corace G, Cerchiara T, Zecchi V. Freeze-dried chitosan/pectin nasal inserts for antipsychotic drug delivery. Eur J Pharm Biopharm. 2010; 75 : 381-87.

LuYan S, Zhen Z, YongHong W, XiaoHua Z. Preparation and application of sulfated konjac glucomannan. J. Food Safety Quality. 2016; $7: 1432-38$

Madgulkar AR, Bhalekar MR, Padalkar RR, Shaikh MY. Optimization of carboxymethyl-xyloglucan-based tramadol matrix tablets using simplex centroid mixture design. J. Pharm. 2013; Article ID 396468, 11 pages.

Mahajan HS, Deshmukh SR. Development and evaluation of gel-forming ocular films based on xyloglucan.Carbo. Polym. 2015; 122: $243-47$.
Mahajan HS, Gundare SA. Preparation, characterization and pulmonary pharmacokinetics of xyloglucan microspheres as dry powder inhalation. Carbo. Polym. 2013; 102 : 529-36.

Mahajan HS' Tyagi VK, Patil RR, Dusunge SB. Thiolatedxyloglucan: Synthesis, characterization and evaluation as mucoadhesive in situ gelling agent. Carbo. Polym. 2013; 91 : 618-25.

Marquez-Escalante J, Carvajal-Millan E, Miki-Yoshida M, Alvarez-Contreras $\mathrm{L}$, et al. Water extractable arabinoxylan aerogels prepared by supercritical $\mathrm{CO}_{2}$ drying. Molecules. 2013; $18: 5531-42$.

Martínez-López AL, Carvajal-Millan E , Rascón-Chu A , Márquez-Escalante J, Martínez-Robinson K. Gels of ferulated arabinoxylans extracted from nixtamalized and non-nixtamalized maize bran: rheological and structural characteristics. CyTA - J. Food. 2013; 11: 22-28.

Martínez-López AL, Carvajal-Millan E, Miki-Yoshida M , Alvarez-Contreras L , Rascón-Chu A, et al. Arabinoxylan microspheres: structural and textural characteristics. Molecules. 2013; 18 : 4640-50.

Mazzarino L, Otsuka I, Halila S, Bubniak L, Mazzucco, S, et al. Xyloglucan-block-poly(E-caprolactone) copolymer nanoparticles coated with chitosan as biocompatible mucoadhesive drug delivery system. Macromol. Biosci., 2014; 14: 709-19.

Mazzarino M, Loch-Neckel G, dos Santos Bubniak L, Ourique F, Otsuka I, et al. 2015. Nanoparticles made from xyloglucan-blockpolycaprolactone copolymers: safety assessment for drug delivery. [ONLINE] Toxicol. Sci. doi: 10.1093/toxsci/kfv114. [Accessed on January 10, 2016].

Md Alam T, Parvez N, Sharma PK. FDA-approved natural polymers for fast dissolving tablets. Hindawi Publishing Corporation J. Pharm. 2014, Article ID 952970, 6 pages.

Mehta DM, Shelat PK, Parejiya PB, Patel AJ, Barot B. Investigations of Plantago ovata husk powder as a disintegrating agent for development of famotidine tablets. Int. J. Pharm. . Nanotechnol. 2011; 4 : 1412-17.

Mikkonen KS. Hemicellulose based blends, composites and nanocomposites in Advances in Natural Polymers: Composites and Nanocomposites. Eds. Thomas S, Visakh PM, Mathw AP. Springer Science \& Business Media, Technology \& Engineering (2012).

Mishra A, Annu Vij Malhotra AV. Graft copolymers of xyloglucan and methyl methacrylate. Carbohydr Polym. 2012; 87 : 1899. 1904.

Mishra RK, Majeed ABA, Banthia AK. Synthesis, characterization and material properties of novel poly vinyl acetate grafted pectin. Int. J. Plastic Technol. 2016; 20 : 79-92.

Morales-Ortega A, Carvajal-Millan E, Brown-Bojorquez F, Rascón-Chu A, et al. Entrapment of probiotics in water extractable arabinoxylan gels: rheological and microstructural characterization Molecules 2014; 19 : 3628-37.

Morris GA, Kok MS, Harding SE, Adams GA. Polysaccharide drug delivery systems based on pectin and chitosan. Biotechnol Genet Engg Rev. 2010; 27 : 257-24.

Nair SB, Jyothi AN, Sajeev MS, Mishra R. Rheological, mechanical and moisture sorption of cassava starch-konjac glucomannan blend films. Starch/Starke. 2011; $63: 728-39$

Namazi H,Fathi F, Heydari A. 2012. Nanoparticles based on modified polysaccharides, the delivery of nanoparticles. Hashim AA(Ed.) ISBN: 978-953-51-0615-9,InTech, Available from: http://www.intechopen.com/books/the-delivery-of nanoparticles/nanoparticles-basedon-modified-polysaccharides.[Accessed on July 14, 2015].

Nayak AK, Pal D, Santra K. Swelling and drug release behavior of metformin $\mathrm{HCl}$-loaded tamarind seed polysaccharide-alginate beads. Int J Biol Macromol. 2015. pii: S0141-8130(15)30032-5. doi: 10.1016/j.ijbiomac.2015.10.027.

Ngwuluka NC, Ochekpe NA, Aruoma OA. Naturapolyceutics: the science of utilizing natural polymers for drug delivery. Polymers 2014; $6: 1312-32$.

Nilsen-Nygaard J, Strand SP, Vårum KM, Draget KI, Nordgård CT. Chitosan: gels and interfacial properties. Polymers. 2015; 7 : 552-79. 
Nino-Medina G, Curvajal-Millan E, Rascon-Chu A, MarquezEscalante JA, Guerrero V, Salas-Munoz E. Feruloylated arabinoxylans and arabinoxylan gels : structure, sources and applications. Phytochem Rev. 2010; $9: 111-20$.

Nisbet DR, Rodda AE, Horne MK, Forsythe JS, Finkelstein DI. Implantation of functionalized thermally gelling xyloglucan hydrogel within the brain: associated neurite infiltration and inflammatory response. Tissue Eng Part A. 2010;16:2833-42.

Nussinovitch A. 2010. Chap 8: Beads as drug carrier in Polymer macro-and micro-gel beads: Fundamentals and applications. Published by Springer Science and Business Media.

Ogaji IJ, Nep EI, Audu-Peter JD. Advances in natural polymers as pharmaceutical excipients. Pharm. Anal. Acta. 2012; $3: 146$.

Pandit AP, Pol VV, Kulkarni VS. Xyloglucan Based in situ gel of lidocaine $\mathrm{HCl}$ for the treatment of periodontosis. Hindawi Publishing Corporation J. Pharm. 2016; Article ID 3054321, 9 pages

Pant S, Malviya R, Sharma PK. Commercialization and biomedical applications of pectin and its formulation in pharmaceutical drug delivery system. Drug Deliv Lett. 2015; 5 : 9-18.

Paz-Samaniego R, Carvajal-Milan E, Brown-Bojorquez F, Rascon-Chu et al. Gelation of arabinoxylans from maize waste watereffect of alkaline hydrolysis conditions on the gel in Wastewater Treatment Engineering. Samed, M. (Ed.). InTech:Croatia. (ISBN: 978953-51-2179-4). DOI: 10.5772/61022. 101-114.

Psyllium website : Available at http://www.psylliums.com/psyllium.htm [Accessed on October 25, 2015].

Rafie F, Jafari B, Yousef Javadzadeh Y. Improving the physicomechanical properties of psyllium husk and evaluation its retarding properties on a water-soluble model drug. Afr. J. Pharm. Pharmacol. 2012; $6: 1124-1130$.

Raja D, Poornima D, Bhaskar K, Saravanan M. Ispaghula huskbased extended release tablets of diclofenac sodium: formulation, evaluation and in vitro release studies. Trop J Pharm Res. 2014; 13 : 205-10.

Rao MRP, Khambete MP, Lunavat HN. Study of rheological properties of psyllium polysaccharide and its evaluation as suspending agent. Int J PharmTech Res. 2011; 3 : 1192-97.

Saeedi M, Akbari J, Morteza-Semnani K, Azarashk A. Evaluation of buccoadhesive propranolol hydrochloride formulations containing Plantago psyllium seed mucilage and Carbopol 934P. Iran. J. Pharm. Sci. 2014; $10: 1-18$.

Silveira JLM, Bresolin TMB. Pharmaceutical use of galactomannans. Quim. Nova. 2011; 34 : 292-99.

Simi CK, Abraham TE. Biodegradable biocompatible xyloglucan films for various applications. Coll. Polym. Sci. 2010; 288 : 297-306.

Singh B, Bala R, Chauhan N. In vitro release dynamics of model drugs from psyllium and acrylic acid based hydrogels for the use in colon specific drug delivery J Mater Sci Mater Med. 2008; 19 : 27712780 .

Singh B, Chauhan N. Dietary fiber psyllium based hydrogels for use in insulin delivery. Int J Diab Mell. 2010; $2: 32-37$.

Singh B. Psyllium as therapeutic and drug delivery agent. Int J Pharm. 2007; $334: 1-14$.

Sumathi S, Ray AR. Release behaviour of drugs from tamarind seed polysaccharide tablets. J Pharm Pharmaceut Sci. 2002; 5:12-18.

Talukder R, Fassihi R. Gastroretentive delivery systems: hollow beads. Drug Develop Ind Pharm. 2004; 30 : 405-12.

Tomczyńska-Mleko M, Brenner $\mathrm{T}$, Nishinari K, Mleko $\mathrm{S}$, Kramek A. Rheological and thermal behavior of mixed gelatin/konjac glucomannan gels. J. Texture Stud. 2014; $45: 344-53$.

Varela MS, Navarro AS, Yamul DK. Effect of hydrocolloids on the properties of wheat/potato starch mixtures. Starch. 2016; 68 : 753-61.

Varshosaz J, Emami J, Tavakoli T, Minaiyan M, et al. Pectin film coated pellets for colon-targeted delivery of budesonide: in-vitro/invivo evaluation in induced ulcerative colitis in rat. Iran. J. Pharm. Res. 2012; $11: 733-45$
Wang J, Yang T, Tian J, Zeng T, et al. Synthesis and characterization of phosphorylated galactomannan: the effect of DS on solution conformation and antioxidant activities. Carbohydr Polym. 2014; $113: 325-35$.

Wang K, Fan J, Liu Y, He Z. Konjac glucomannan and xanthan gum as compression coat for colonic drug delivery: experimental and theoretical evaluations. Front Chem Engg. 2010; 4 : 102-108.

Watts P, Smith A. PecSys: in situ gelling system for optimised nasal drug delivery. Expert Opin Drug Deliv. 2009; 6 : 543-52.

World Health Organisation (WHO) Food additive series No. 5. Toxicological evaluation of some food additives including anti-caking agents, antimicrobials, antioxidants, emulsifiers and thickening agents. Available at: http://www.inchem.org/documents/jecfa/jecmono/ v05je82.htm [Accessed on September 22, 2015].

World Health Organisation (WHO) Food additive series. Toxicity profile of konjac flour. Available at: http://www.inchem.org/documents/jecfa/jecmono/v32je10.htm [Accessed on December 14, 2015].

Wu J, Deng X, Lin X. Swelling characteristics of konjac glucomannan superabsorbent synthesized by radiation-induced graft copolymerization. Radiation Phys. Chem. 2013; 83 : 90-97.

Xia B, Ha W, Meng XW, Govender T, et al. Preparation and characterization of a poly(ethylene glycol) grafted carboxymethyl konjac glucomannan copolymer. Carbohydr. Polym. 2010; 79 : 648-65.

Xiang Z, Runge T. Emulsifying properties of succinylated arabinoxylan-protein gum produced from corn ethanol residuals. Food Hydrocoll. 2016; 52 : 423-430.

Xiao C, Gao S, Wang H, Zhang L. Blend films from chitosan and konjac glucomannan solutions. J Appl Polym Sci. 2000; 76 : 509-515.

Xiao M, Dai S, Wang L, Ni X, Yan W, et al. Carboxymethyl modification of konjacglucomannan affects water binding properties. Carbo. Polym. 2015; $130: 1-8$.

Xiao M, Dai S, Wang L, Ni X, et al. Carboxymethyl modification of konjac glucomannan affects water binding properties. Carbohydr Polym. 2015;130 : 1-8.

Yadav G, Sharma N, Mayank Bansal M, Thakur N. Application of natural polysaccharide for delivery of biopharmaceuticals. Int J Pharm. Life Sci. 2013; 4 : 2756-65.

Yan Y, Takemasa M, Zhao C, Yu L, Nishinari K. Structuregelation research on gallate analogs and xyloglucan by rheology, thermal analysis and NMR. Food Hydrocoll. 2016; 52 : 447-59.

Yao K, Li J, Yao F, Yin Y. Chitosan-Based hydrogels: functions and applications. CRC Press, 2016.

Yu H, Huang A, Xiao C. Characteristics of konjac glucomannan and poly(acrylic acid) blend films for controlled drug release. J. Appl. Polym. Sci. 2006; 100: 1561-1570.

Zhang YQ, Gan X, Li XL, Xie B. In vitro static adsorption behavior of sulfated konjac glucomannan gel beads. 2008 2nd International Conference. on Bioinformatics and Biomedical Engineering . 2008; $1531-34$.

\section{How to cite this article:}

Majee SB, Avlani D, Biswas GR. Non-Starch Plant Polysaccharides: Physicochemical Modifications and Pharmaceutical Applications. J App Pharm Sci, 2016; 6 (10): 231-241. 